\title{
The Methodological Approach to the Co-Creation of Online Health Education with and for Individuals with Intellectual Disability
}

\section{Get Wise About Your Health Supporting People with Intellectual Disability to be Health Savvy}

\author{
Burke $\mathrm{EA}^{1 *}$, Dennehy $\mathrm{H}^{1}$, Bakker $\mathrm{AR}^{2}$, Bowman $\mathrm{S}^{3}$, Murphy $\mathrm{E}^{1}$, Maes-Festen $\mathrm{D}^{2}$, McCallion $\mathrm{P}^{4}$, McCarron $\mathrm{M}^{1}$ and \\ Oppewal $\mathrm{A}^{2}$
}

${ }^{1}$ Trinity Center for Ageing and Intellectual Disability, School of Nursing and Midwifery, Trinity College Dublin, The University of Dublin, Dublin 2 ${ }^{2}$ Department of General Practice, Intellectual Disability Medicine, Erasmus MC, University Medical Center Rotterdam, Rotterdam, The Netherlands ${ }^{3}$ Office of the Dean of Research, Trinity College Dublin, the University of Dublin, Dublin 2

${ }^{4}$ Temple University College of Public Health, Philadelphia, USA

Submission: March 11, 2021; Published: March 23, 2021

*Corresponding author: Burke EA, Trinity Center for Ageing and Intellectual Disability, School of Nursing and Midwifery, Trinity College Dublin, The University of Dublin, Dublin 2

Abstract

Background: Health inequalities are evident among adults with intellectual disability. Two large national longitudinal studies have highlighted the paucity of health education among people with intellectual disability. Frequently people with intellectual disability are excluded from health screenings, targeted health promotions and health education. Subsequently health problems often go unrecognised and unmet.

Aims: Through co-creation and collaboration, the aim of this project was to develop, refine and validate resources to deliver health education on a novel digital platform in an accessible easy-read manner. The aim of this paper is to describe how the project engaged individuals online to co-create this educational offering.

Methods: This is a collaborative project between Ireland and the Netherlands. The project adopts a strengths-based approach and the design adopted is citizen co-creation to promote citizen engagement and involvement in the development of this novel online programme. Individuals with intellectual disability, their carers and researchers worked in partnership to develop content, and co-design the platform. This was enabled by building familiarisation with online engagement with individuals with intellectual disability, designing and developing easy-read accessible materials and through the implementation of a robust consistent approach in both countries.

Results: With the appropriate supports in place individuals with intellectual disability were capable of engaging productively and successfully with online interviewing. Many participants had experience using the internet however for many this was their first time engaging online in such a process. In total 37 participants successfully completed the online interviews and pilot-testing, contributing to the development of the content and digital education platform.

Conclusion: The digital platform supports individuals with intellectual disability to be better prepared for engaging with healthcare professionals. The aims are increased confidence, and better understanding of what one can do to look after one's health. The process for developing the co-created materials identifies four critical areas for a successful project or programme: 1) establishing or utilizing relationships where a high degree of trust is in place; 2) assessing technology access and literacy levels across platforms; 3) providing skills training or other support to ensure contributors are actively and meaningfully involved across the project's lifecycle; and 4) ensuring feedback is acknowledged and used in the co-creation of both process and summative deliverables.

Keywords: Accessibility; Co-creation; Digital platform; Health education; Intellectual disability; Online learning; Internet usage

\section{Introduction}

People with intellectual disability are living longer however continue to experience significant health disparities and earlier mortality than their peers in the general population $[1,2]$. Contributing factors to this include: a predisposition to chronic 
health conditions; diagnostic overshadowing; limited access to targeted health promotions and inaccessible health information $[3,4]$. Overall people with intellectual disability access healthcare at higher levels than the general population, however despite this, greater levels of chronic health and multimorbidity persist [5-7]. Rates of chronic disease are overall higher for individuals with intellectual disability in comparison to the general population $[4,8]$. One condition that is highly prevalent within this population is osteoporosis [9-11]. Research has sought to explore and understand the prevalence for poor bone health among people with an intellectual disability and prevalence rates of over $70 \%$ have been identified [12]. Research has also highlighted the low levels of doctor's diagnosis of osteoporosis among older adults with intellectual disability, despite reported high prevalence of risk factors, which greatly impacts health outcomes [13]. Compounding these health disparities and difficulties are high rates of illiteracy and communication challenges [14].

Global health policies advocate for citizen responsibility for their health and advance a vision of creating an environment where individuals are valued and enjoy health and wellbeing as they age $[15,16]$. This is challenging for people with intellectual disability considering the high rates of illiteracy, diverse cognitive limitations, as well as reported anxiety and difficulty when communicating with healthcare professionals. Currently, there are no nation-wide health promotional campaign activities specifically designed or targeting people with intellectual disability, further impacting negatively on their health experience. In addition, health educational programmes for people with intellectual disability do not always address the health disparities actually experienced by people with intellectual disability [17]. Furthermore, when access to healthcare programmes is considered it is often the physical access that comes to mind; however, for people with intellectual disability access is far more complex. Education, healthcare information and health promotion materials that are easy read, accessible built in partnership with individuals with intellectual disability is what is required. All too frequently programmes are developed without the input of individuals with intellectual disability and healthcare staff lack the ability to stimulate involvement [18]. There is often a lack of evidence about specific and broad health needs for people with intellectual disability [3], and subsequently those developing guidelines to improve health for all, can unintentionally increase health inequalities for people with intellectual disability due to the lack of their engagement and involvement [19]. Additionally, there is limited evidence on the use or uptake of e-health technology among ageing adults with intellectual disability, and as a result, research has recognised the need for the development of e-health interventions for older adults with intellectual disability [20]. Targeted health interventions are essential for adults ageing with intellectual disability, to improve health and well-being outcomes, as well as their knowledge so as to be empowered to engage in meaningful self-care and skill-development opportunities.
Recognising these deficiencies and in alignment with the principles of the 2030 Agenda for Sustainable Development (Goals 4, 8, 10 and 17) [21] as well as United Nations Educational, Scientific and Cultural Organization (UNESCO, 2005), which advocate education for all citizens, the 'Get Wise about your health' project has been co-created and co-developed with people with intellectual disability and their carers. The principle aims of the project being to address significant health issues by providing accessible health education for people with intellectual disability. Embedded in an emancipatory philosophy for conducting research and from previous experience [3], empowering individuals with opportunity to engage yields meaningful results. Get Wise about your health, is a European project funded by EIT Health, and is a partnership between Trinity College Dublin and Erasmus MC, University Medical Center Rotterdam. The project offers excellence in best practice health promotion education for people with intellectual disability, encapsulating citizen involvement and building on the strengths of the core partners. Providing accessible health education promotes health awareness and ultimately improved health outcomes for individuals with intellectual disability [22]. The aim of this paper is to describe the methodological aspects of developing the project and how the team engaged and involved individuals online to co-create the Get Wise educational offering.

\section{Methods}

\section{Project Aim and objective}

The aim of 'Get Wise about your health' was to develop, refine and validate a health education programme in collaboration with individuals with intellectual disability in an accessible, easy to read manner on a novel digital platform. The objective was to address and improve individual's health knowledge to empower the individual to become an informed consumer of health.

\section{Project Design}

The project was initially designed to engage individuals in a face-to-face manner, however, due to the emergence of COVID19 the engagement model changed to a totally online experience. Get Wise stayed true to its philosophy of emancipatory research employing a strengths-based approach in the design of the project, whereby individuals with intellectual disability, their carers and researchers worked in partnership to develop content, contribute, and refine materials and validate resources through the following activities:

1. Building familiarisation with online engagement,

2. Designing and developing easy-read accessible materials,

3. Co-creation, review and validation of all modules and content,

4. Co-creating the accessible educational online platform. 
The team encouraged familiarisation through the recruitment process, building connections and support for individuals through the services networks to enable online engagement. Consistent with European guidelines, easy read information was developed [23], reviewed by individuals and finalised to support understanding and the roll out of the project. The educational online platform and all easy read material were developed in collaboration with adults with intellectual disability. To inform the content the project adopted a qualitative approach through semistructured interviews using an online questionnaire with openended questions addressing going to the doctor and bone health. To ascertain the participants' online engagement, individuals were also asked about their experience using technology; they were asked if they had devices and what they used them for, if they had access to the internet, and if they needed support to facilitate access and involvement. Once the information from the online interviews had been collated, this informed both the supports required to facilitate ongoing engagement and the development of the content within the modules of the online accessible health education programme. A separate group of participants were invited to participate in a pilot study once the platform had been developed, to test the level of accessibility of the programme, the user friendliness of the platform and the feasibility of the tools developed. This allowed for reasonable adjustments and amendments to be applied to the platform.

\section{Project Population}

The population includes adults with mild and moderate intellectual disability. Participants were recruited from existing client panels and those specifically linked to the larger longitudinal studies in both Ireland and the Netherlands, IDSTILDA (The Intellectual Disability Supplement to the Irish Longitudinal Study on Ageing) and HA-ID (Healthy Aging and Intellectual Disabilities). Client panels were approached through established contacts from the TCAID (Trinity Centre for Ageing and Intellectual Disability) academic collaborative centre and the HA-ID consortium, to establish if they had individuals who were in good health in light of the current circumstances (COVID19 pandemic), and if they would like to participate in the project. The project recruited 20 participants for online interviews and an additional 17 participants for pilot-testing across both countries. The team also engaged with five general practitioners in Ireland and the Netherlands to enrich the data collected from people with intellectual disability, and further support the content development of the Get Wise course modules.

\section{Recruitment and building familiarity}

Recruitment for the project began in April 2020, initially recruiting for the interviews and then separately for the pilot study. Three service providers in Ireland and three in the Netherlands were identified as potential project sites. Each service provider's
Director was approached by the Principal Investigator (PI) to establish their interest in the project and confirm the presence of individuals who would be in a position to provide their opinion on the proposed project. In Ireland, services were then contacted by email, with details on the 'Get Wise about your health' project and, upon further confirmation of interest from the service, an application to the ethics committee of each service was submitted. On successfully gaining ethical approval, permission was sought from the Director of the service to identify individuals within the service who may be interested in collaborating on the project. In the Netherlands, the service providers were first asked whether they were interested in participating, after which an application to the ethics committee of the Erasmus MC, University Medical Center Rotterdam was submitted. After gaining ethical approval, the Board of Directors of the service providers gave formal permission for collaboration in the project, and individuals that could participate were identified via client panels. In both countries, the PI working with the research assistant (RA) contacted the individuals and their key worker. They were invited to discuss their involvement in the project online or by phone, and subsequently invited to contribute to the project. On confirmation of an expression of interest, an individual information pack was sent to each person via email or post and the key worker was contacted to assist with downloading and supporting understanding. This information packet consisted of an accessible invitation letter, an accessible information booklet, and an accessible consent form. The team in Ireland also created a QR code link which was included in the information pack. The participant could scan the QR code to hear and see the RA sign (Lámh) the information about the project. Lámh is a sign system used in Ireland, by children and adults with intellectual disability and communication needs (see: https:// www.lamh.org/). This contributed a further aspect to promoting and enabling accessibility as well as building the individual's familiarity with the project and the project team.

The individuals were asked to consider the project and return their consent to become involved in its co- creation. On receipt of the consent forms, the project team arranged a date and time to complete the interview. All contact information for the PI and researchers was provided, should the individual have any queries or questions. The individuals were given a minimum of seven to fourteen days to consider the information. During this process the project team contacted the keyworker only and no personal participant information was taken or held by the project team. A total of 20 participants were recruited to conduct the online interviews across both countries.

Additional service providers were approached to support pilot testing the programme on its completion. In total 17 participants were recruited from existing client panels and the recruitment process followed the same process as recruitment for online interviews. 


\section{Inclusion criteria}

- $\quad$ Adults with an intellectual disability

- $\quad$ Self-advocating by self-declaration

- $\quad$ Capacity to consent to take part in this project

- Consents to inclusion and participation in the project

\section{Exclusion criteria}

- $\quad$ Children or young adolescents

\section{Withdrawal of Participants from the Project}

Participants were assured of their rights at the beginning of each interview and could withdraw from the study at any point. They were not required to specify a reason for withdrawal, nor were there any consequences for doing so. Participants were reminded of their rights through a system of process consent during the interview and their choice was always respected. Participants were encouraged to take a break at any point during the interviews to ensure they were not overburdened. Furthermore, if the participants wished, their keyworker could be present throughout the interview and had the discretion to withdraw a participant from the project for a medical reason if they deemed this necessary. The team sought the keyworker's assistance to ensure all potential participants understood the project and were aware of their right to participate or not without any negative consequences from their choice.

\section{Follow-Up of withdrawal participants}

If participants wished to withdraw from the project the protocol was to provide them with an opportunity to speak with the researcher to discuss any concerns or questions they may have had. When needed, the participant's key worker was to be present for the interview to ensure that adequate support to the individual could be provided. A debrief sheet would also be provided to the participant, and the PI and RA would go through this in detail with each participant. This included relevant contact information and details of appropriate supports, should the participant require. No participant withdrew during the project.

\section{Procedure}

Following the change of project implementation due to COVID19 impact, participants were invited to conduct the interview through video call (MS Teams) or by telephone, with the researcher. The PI and RA held an information session with each individual, prior to the interview, detailing the project protocol and building familiarisation with the online meeting system. The PI and RA also went through the informed consent process and ensured that consent was obtained. The interviews lasted 20-45 minutes in total. Participants completed the interview at a time and place of their choosing over video call with the support of their keyworker when needed. Choice of conducting a phone call or video call was offered however all participants opted for a video call. Agreement to also have interview audio recorded was confirmed. The questionnaire included three sections: 1 . Attending the Doctor; 2. Bone Health; 3. Digital Use. All questions were presented in easy read format, plain language supported with visual representation. As the project is focused on improving people's knowledge and experience of health, all questions were posed positively and focused on best practice. Knowledge gaps were identified to inform module content and participant expertise was sought to inform the overall accessibility and interface of the programme. Each keyworker played a central role in assisting with the interviews. Once the interview was complete, the researcher went through the debrief form with each participant and the participant had the opportunity to discuss the interview and ask any further questions that they may have had. All data gathered from the interview was transcribed by the RA.

\section{Ethical Considerations}

\section{Consent}

On receipt of the consent the interview was arranged. Before each interview began, the team went through the nature of the questions which would be asked during the interview. Participants were informed that any information discussed throughout the interview was private and confidential. The individual was asked to sign or mark (if unable to sign their name) the consent form. The mark made by the participant was witnessed by their keyworker, to confirm that they had consented to participate.

\section{Photography/Video}

The project sought permission to photograph the online interviews, with the purpose of demonstrating the process in action and as evidence of work complete for the purposes of audit from the funders. This was identified in the consent form and the information booklet. Any individual who did not consent to having their photo taken had their right of choice respected and protected.

\section{Risk Management}

Get Wise was a limited risk project, however as people were asked to give their opinion on personal experiences and information, there was the potential that discussion of these topics could unearth unpleasant prior experiences or memories of a challenging period of time that the participant had experienced. To manage this risk, the research team enacted a range of strategies to appropriately respond to any potential distress. These included consultation with participants regarding stopping the interview, taking a break or redirection, and changing of topic. The researchers also worked with each keyworker to ensure the utmost safety of all participants. The researchers were experienced in working with and supporting people with intellectual disability; they were trained in sensitive interviewing and in responding appropriately to participants in distress. 


\section{Debrief \& Follow-Up}

Participants were provided with a debrief sheet in easy read format. The PI and RA checked through this with each participant after the interview. This provided an opportunity for the participant to ask any further questions and reflect on their experience. A specified email was provided for the participant to contact the PI, should they have any additional questions after the interview had been completed.

\section{Data Management Plan}

\section{Participant Information}

All data was handled confidentially, and each participant received a numeric, four-digit code, which was not related to initials, birth date, or the code of the services. Data collected, entered, and analyzed was pseudonymized. Only the researcher team had access to the source data in which the codes were linked to the individual participant. The PI and RA had access to the signed consent forms and securely kept all signed consent forms separate from the interview transcripts, for the life of the project plus the statutory years in accordance with data protection legislation in each country. All data is stored in encrypted secured drives in each respective universities. Participants were asked their name for the purposes of engaging with the project team during the online interview but no other personal information such as age, address or current status was requested.

\section{Handling \& Storage of data and documents}

Data from the interviews was transcribed verbatim from a recording of the online interview on MS teams, no individual outside of the project team has access to the information collected. No copies of the information collected were allowed. The information collected was themed and only the themes emerging from the analysis of the discussion were shared to inform the development of the module content. Vignettes or quotes were used; however, these have been anonymised.

\section{Data Analysis}

The interview data-analysis began after the first interviews and was executed simultaneously with the data-collection. Thematic analysis was used to analyse the transcripts, using the six-step Braun \& Clarke [24] framework. The first two Irish interviews were analyzed by two researchers. Together they constructed a coding-tree. Initially this was achieved by giving open codes to the data. These codes were then grouped into overarching themes. Any differences of opinion between the researchers were resolved through discussion. Once the coding tree had been constructed the researchers coded the data separately. A similar process was employed in the Netherlands. When the data had been coded, the researchers discussed the results with the wider team and constructed final themes across both sets of data. For the purposes of this paper the results of this section of the project will not be reported here.

\section{Interface review}

On completion of the building of the digital platform and first two modules online, 'Get Wise About Visiting the Doctor' and 'Get Wise About Your Bone Health', a new group of participants were recruited from existing client panels, linked to longitudinal studies in both Ireland and the Netherlands to pilot-test the programme. This ensured that any potential bias was avoided. The link to the digital platform was shared with services who had identified individuals who were interested in pilot-testing the programme. A pilot- test guide was distributed to interested individuals and their keyworkers, which included instructions about how to use the website and work through the modules. The guide also contained a list of sample questions relating to the course content and website interface. Participants were invited to provide feedback on the course content and accessibility of the platform. The RA in both countries liaised with keyworkers and participants through phone calls and email, to compile this feedback. This allowed for reasonable adjustments to be made by the graphic and web designers, before the accessible digital platform was launched. Feedback provided referenced difficulty with finding the button for Dutch translation, difficulty with finding the transcript button, scaling issues and difficulty with accessing the downloadable tools and certificate.

\section{Results}

In total, 37 participants engaged in the project from both Ireland and the Netherlands. The team and participants experienced equal challenges and success during the data collection period. Direct support, building familiarity, good communication with service providers and ensuring the research team were available for questions, contributed to overcoming many of the challenges and to the overall success of the project. For many participants, the online interview with the research team was the first time they had engaged with people outside of their support bubbles and families, since the emergence of COVID-19.

\section{Engagement with technology}

The majority of participants reported a high uptake of using technology, with most participants disclosing that they would like to engage and use technology more often. In both countries, most participants $(91.8 \%)$, reported that they used the internet often. In the Netherlands, all participants but one had previous experience with video calls. All participants in Ireland required support with setting up the video call. Out of the Dutch participants, 4 received support of keyworkers in setting up the video call and 5 participants did not need support. Participants across both countries used a range of technology to access the internet, including laptops, Smartphones, and tablets (Figure 1). All participants in the Netherlands had access to the internet, while $75 \%$ of participants had access to the internet in Ireland. However a number of people (17.9\%) in Ireland were unsure as to whether they had access to the internet with $7.1 \%$ reporting they did not have access to the internet. A breakdown of both countries is provided below (Figure 2). 


\section{Global Journal of Intellectual \& Developmental Disabilities}

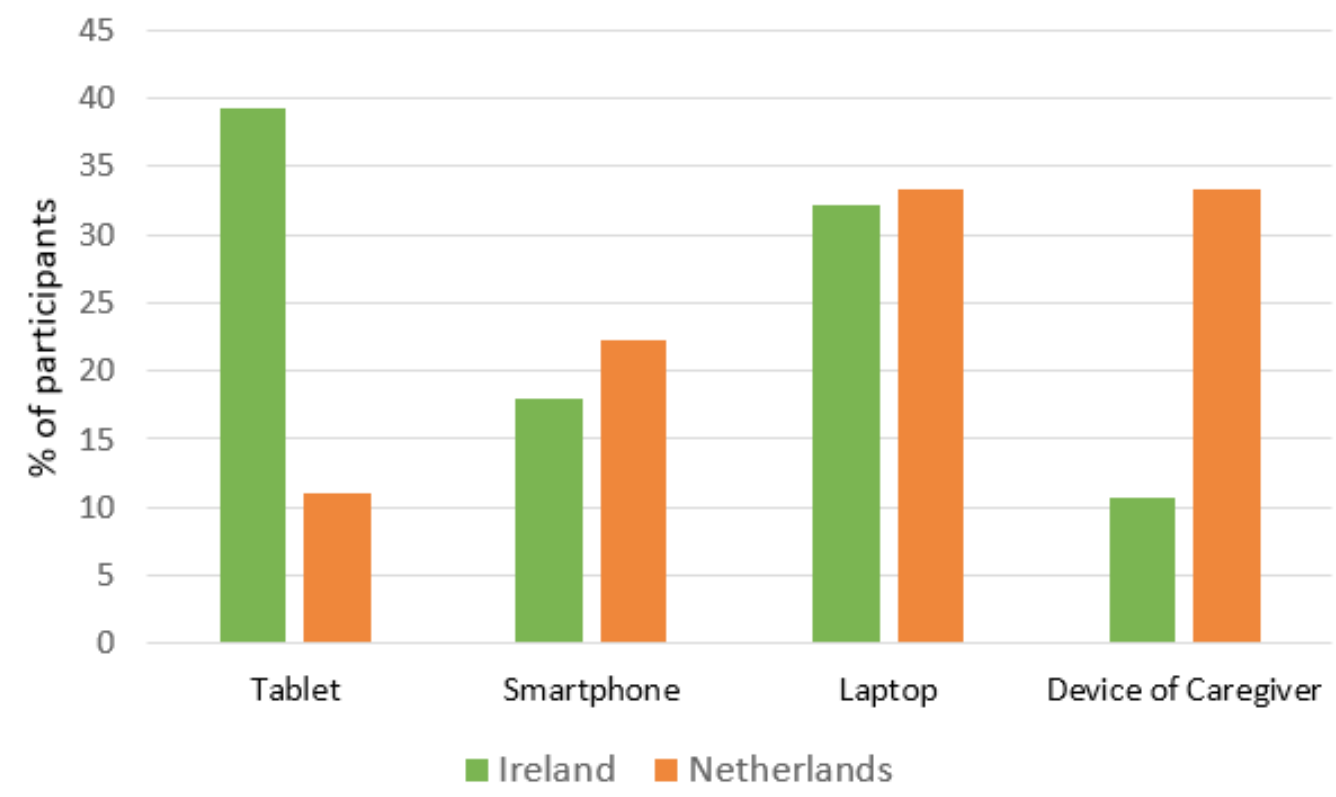

Figure 1: Technology used to access the internet.

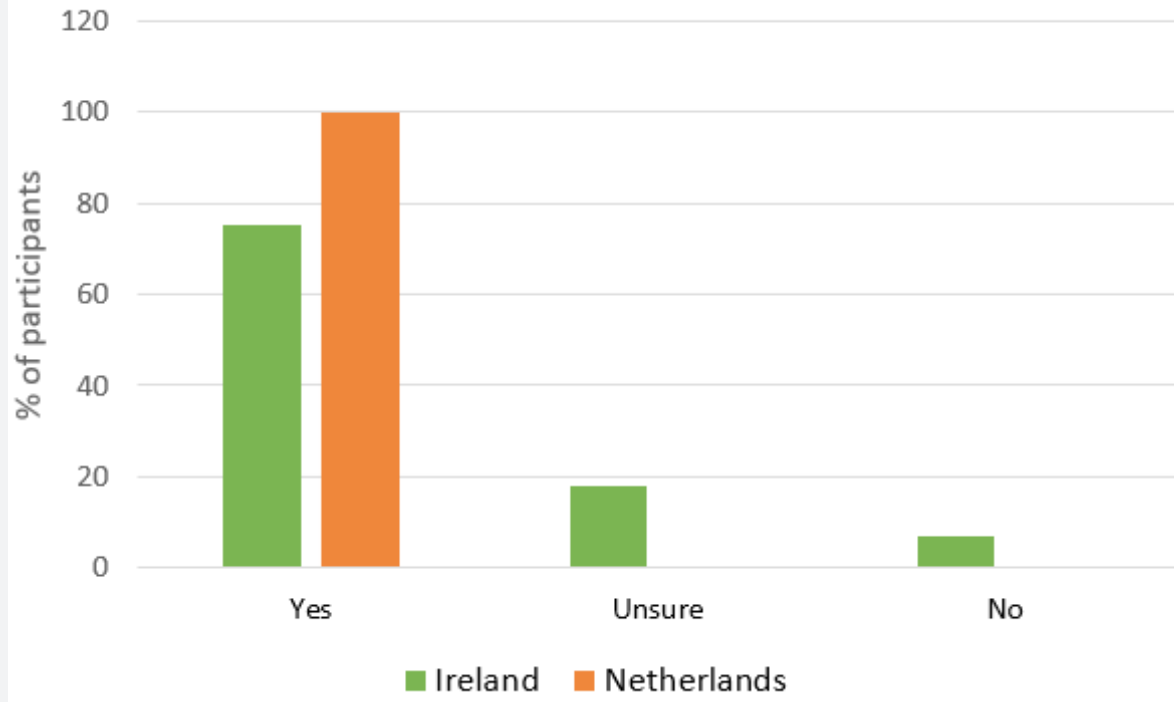

Figure 2: Access to the internet.

\section{Enabling engagement in the interview process}

All interviews were organized at a time that suited the participant's schedule. Each interview was supported with easy read accessible information in advance. Participants found this very helpful as they noted the material allowed them to prepare and plan for the day of the interview and allowed them to consider questions, they may have for the project team. The accessible material also enabled them to understand what the process would entail, how long it would be, who exactly would see them and their information. It also provided clear instruction on their rights and who they could have with them, which they reported they found respectful.

Initially there were challenges with internet connections or access to the internet which meant some scheduled interviews 
had to be reset. However, participants were supported to travel to their service, when appropriate, to gain online access. During data collection COVID-19 restrictions changed in both countries and this did pose problems, such as the burden the lockdown measures posed on the personal life of participants, which were overcome with detailed planning and support from keyworkers, carers and the service providers. One participant withdrew from the study due to the burden of restrictions on their personal life, and they experienced difficulty navigating these very difficult circumstances and participating in the pilot. Participants in Ireland required support with setting up the online call, while just over half of participants in the Netherlands did not require support to do this. Keyworkers and service providers played a vital role in enabling this. Facilitating participants to engage online also had the additional benefit of limited disruption to their day and it meant participants had limited travel requirements. Participants were also able to offer immediate feedback on their experience to both their advocacy groups and the project team. While participants responded positively to the online interviews and spoke openly with the research team and their keyworkers, they reported that they would still prefer to meet in person. Participants reported that they were looking forward to a time when interviews could be conducted in-person again and referenced the informal chat and tea which would usually occur after an interview. However, they did note that they now used the internet to stay in contact with their family.

Examining the feedback from participants regarding the online educational platform, participants demonstrated that overall, they have a high level of competence when using the internet and apps. Participants reported a high uptake of (64.9\%) online or computer training within their services in Ireland (64.86\%), which has been reported as a positive experience for all participants. Participants also reported a preference for interactive learning, with support from their keyworker, which they could complete over an extended period of time. Participants recommended that a guide be developed to support the learner as they work through the platform and modules. Subsequently the team have developed an orientation guide which is available on the platform.

\section{Discussion}

Online interviews were a novel method of data collection with people with intellectual disability and one that was not initially planned for this project. However, it proved interesting to record participants experiences of engaging in online research, as well as their knowledge and experience of engaging with technology. Promoting an inclusive approach to the research ensured meaningful engagement and optimized the experience for the participants which Schwartz et al. [25] note is an essential part of promoting good collaboration and strengthened this project's philosophy of co-creation and co-production. Some participants reported that while they had enjoyed the experience, it was important for them to know the research team prior to conducting the online interviews. This was facilitated by building familiarity through online information meetings and ensuring the project team's photos were included in the accessible material with the added benefit of offsetting any potential communication barriers that may arise. With familiarity it was noted the individual's confidence grew and their engagement in the process reaped dividends for the team. This familiarity promotes partnership between the researcher and the participant establishing interest and benefit for both parties which Schwartz and colleagues [25] note are components that will over time improve the process and maintain collaboration. Furthermore, it is agreed that working with individuals, providing a variety of communication styles and improving collaboration will ultimately promote the best possible outcome for individuals with intellectual disability who heretofore were excluded from the research process [26-28].

There were certain challenges associated with conducting online interviews such as ensuring the necessary supports were available and scheduling. There has been a recent growth, particularly with the onset of the pandemic, of internet use among those with intellectual disability and it has been shown that with the correct support and guidance individuals can engage online [29] which was the case in this project. However, broadband and device access proved to be the greatest challenge in this project. Lack of broadband, lack of devices and poor connectivity combine to impede individual's digital ability and growth. While many countries strive to provide more and faster internet access there are pockets, particularly in rural areas, in most countries that continue to suffer from inadequate access contributing to a digital divide that can ultimately contribute to social exclusion and impact negatively on the economy [30]. For individuals with intellectual disability this contributes further to the challenges they experience when trying to engage with social media and technology [31]. If these issues of access are not addressed, it will further contribute to marginalizing individuals and increase the division between those who can and those who cannot access the internet. In this project the key to overcoming such challenges was the support individuals received from their carers and service providers. It was also evident that participants had a high level of competency with technology and used technology throughout their daily lives. What this project has identified is that providing the right opportunity and circumstance, and with the right supports in place individuals with intellectual disability can use and engage successfully online. Further, they can be involved in research and in co-creating health promotional materials and resources. This lesson is transferable to all online engagements not just research. It is hoped that this message will emphasise ability and dispel the perceived notion that people with intellectual disability cannot use or access the internet [32]. For those who participated in this project they reported that they used technology to connect with family, friends and social groups, and to engage with education at various levels, as well as using technology for entertainment 
purposes, shopping and to gather more information on topics of interest. It is evident that technology increases accessibility to a wide range of activities, communities, experiences and people. Individuals with intellectual disability want to have the same experiences and opportunities as everyone else [33]. This project demonstrates that this is possible. Overall, participants reported that they would like to engage with technology more often and to incorporate it more into their daily lives and routines.

\section{Conclusion}

Online interviews in this project provided a method in which people with intellectual disability were able to share their expertise, knowledge and experiences of health education and health provision, which has contributed to the development of the digital health education platform, Get Wise about your Health. This platform addresses the health disparities experienced by people with intellectual disability and offers an accessible and practical solution which has been co-designed and co-developed by people with intellectual disability through the online platform. Despite the inference in the literature and the perceived idea that people with intellectual disability are not capable, given the right supports and access to opportunities and technologies engaging online is possible. Overall participants reported their online interviews as being very positive and the rich data collected is testament to that. Participant engagement was extremely high, and the knowledge and personal experiences gathered from participants greatly contributed to the development of the module content and key learnings within the course developed. Most participants reported online interviews as a positive experience, which they would be happy to participate in again. Engaging individuals with intellectual disability online was a very successful method of data collection. The Get Wise course is available online in both English and Dutch and can be found at www.getwiseid.eu.

\section{Acknowledgements}

The authors would like to sincerely thank the participants, their families, the General Practitioners, the key workers and service providers in both Ireland and the Netherlands for their contributions to and support of the Get Wise project. Acknowledgment and thanks is also extended to our funders EIT Health. Grateful appreciation is extended to our team of animators, graphic designers and web developers.

\section{References}

1. McMahon M, Hatton C (2021) A comparison of the prevalence of health problems among adults with and without intellectual disability: A total administrative population study. Journal of Applied Research in Intellectual Disabilities 34(1): 316-325.

2. McCarron M, Carroll R, Kelly C, McCallion P (2015) Mortality rates in the general Irish population compared to those with an intellectual disability from 2003 to 2012. Journal of Applied Research in Intellectual Disabilities 28(5): 406-413.

3. Burke ÉA, Walsh JB, McCallion P, McCarron M (2020) Making reasonable adjustment to enable and support people with intellectual disability engage in objective health measures in a research studythe health fair in the intellectual disability supplement to the Irish longitudinal study on ageing. Inclusion 8(2): 124-137.

4. McCarron M, Cleary E, McCallion P (2017) Health and healthcare utilization of the older population of Ireland: comparing the intellectual disability population and the general population. Research on aging 39(6): 693-718.

5. Hussain R, Wark S, Janicki MP, Parmenter T, Knox M (2020) Multimorbidity in older people with intellectual disability. Journal of Applied Research in Intellectual Disabilities 33(6): 1234-1244.

6. Hermans H, Evenhuis HM (2014) Multimorbidity in older adults with intellectual disabilities. Research in Developmental Disabilities 35(4): 776-783.

7. McCarron M, Swinburne J, Burke E, McGlinchey E, Carroll R et al. (2013) Patterns of multimorbidity in an older population of persons with an intellectual disability: results from the intellectual disability supplement to the Irish longitudinal study on aging (IDS-TILDA) Research in developmental disabilities 34(1): 521-527.

8. De Winter CF, Bastiaanse LP, Hilgenkamp TIM, Evenhuis HM, Echteld MA (2012) Cardiovascular risk factors (diabetes, hypertension, hypercholesterolemia and metabolic syndrome) in older people with intellectual disability: results of the HA-ID study. Research in developmental disabilities 33(6): 1722-1731.

9. Frighi V, Morovat A, Andrews TM, Rana F, Stephenson MT, et al. (2019) Vitamin D, bone mineral density and risk of fracture in people with intellectual disabilities. Journal of Intellectual Disability Research 63(4): 357-367.

10. Burke ÉA, Carroll R, O’Dwyer M, Walsh JB, McCallion P, et al. (2018) Osteoporosis and People with Down Syndrome: A Preliminary Descriptive Examination of the Intellectual Disability Supplement to the Irish Longitudinal Study on Ageing Wave 1 Results. Health 10(9): 1233-1249.

11. Bastiaanse LP, Mergler S, Evenhuis HM, Echteld MA (2014) Bone quality in older adults with intellectual disabilities. Research in developmental disabilities 35(9): 1927-1933.

12. Burke ÉA, Carroll R, O’Dwyer M, Walsh JB, McCallion P, et al. (2019) Quantitative examination of the bone health status of older adults with intellectual and developmental disability in Ireland: a cross-sectional nationwide study. BMJ open 9(4): e026939.

13. Burke ÉA, McCallion P, Carroll R, Walsh JB, McCarron M (2017) An exploration of the bone health of older adults with an intellectual disability in Ireland. Journal of Intellectual Disability Research 61(2): 99-114.

14. Smith M, Manduchi B, Burke É, Carroll R, McCallion, et al. (2020) Communication difficulties in adults with Intellectual Disability: Results from a national cross-sectional study. Research in developmental disabilities 97: 103557.

15. World Health Organisation (2020) The United Nations Decade of Healthy Ageing.

16. Department of Health (2013) Healthy Ireland, A Framework for Improved Health and Wellbeing 2013- 2025. Government of Ireland.

17. Doody CM, Doody O (2012) Health promotion for people with intellectual disability and obesity. British Journal of Nursing 21(8): 460-465.

18. Pelleboer-Gunnink HA, Van Oorsouw WMWJ, Van Weeghel J, Embregts PJCM (2017) Mainstream health professionals' stigmatising attitudes towards people with intellectual disabilities: A systematic review. Journal of Intellectual Disability Research 61(5): 411-434.

19. Cooper SA, Melville C, Morrison J (2004) People with intellectual 
disabilities: their health needs differ and need to be recognised and met. BMJ: British Medical Journal 329(7463): 414.

20. Vázquez A, Jenaro C, Flores N, Bagnato MJ, Pérez MC, et al. (2018) E-health interventions for adult and aging population with intellectual disability: a review. Frontiers in psychology 9: 2323.

21. United Nations 2015 Transforming our World: The 2030 Agenda for Sustainable Development. Available at: https://sdgs.un.org/ publications/transforming-our-world-2030-agenda-sustainabledevelopment- 17981 .

22. Cardell B (2015) Reframing health promotion for people with intellectual disabilities. Global qualitative nursing research 2: 2333393615580305.

23. Inclusion Europe (2015) Information for all. European standards for making information easy to read and understand. Education and Culture DG. Lifelong learning programme. https://inclusionireland.ie/ wp- content/uploads/2020/11/european-easy-read-standards.pdf.

24. Braun V, Clarke V (2006) Using thematic analysis in psychology. Qualitative Research in Psychology 3(2): 77-101.

25. Schwartz AE, Kramer JM, Cohn ES, McDonald KE (2020) "That felt like real engagement": Fostering and maintaining inclusive research collaborations with individuals with intellectual disability. Qualitative health research $30(2): 236-249$.

26. Chew KL, Iacono T, Tracy J (2009) Overcoming communication barriers: Working with patients with intellectual disabilities. Australian family physician 38(1/2): 10 .
27. Brooker $\mathrm{K}$, van Dooren $\mathrm{K}$, Tseng $\mathrm{CH}$, McPherson L, Lennox N, et al. (2015) Out of sight, out of mind? The inclusion and identification of people with intellectual disability in public health research. Perspectives in public health 135(4): 204-211.

28. Di Lorito C, Bosco A, Birt L, Hassiotis A (2018) Co-research with adults with intellectual disability: A systematic review. Journal of Applied Research in Intellectual Disabilities 31(5): 669-686.

29. Näslund R, Gardelli Å (2013) 'I know, I can, I will try': youths and adults with intellectual disabilities in Sweden using information and communication technology in their everyday life. Disability \& Society 28(1): 28-40.

30. Hampton K, Fernandez L, Robertson C, Bauer JM (2020) Repercussions of Poor Broadband Connectivity for Students in Rural and Small Town Michigan. SSRN 3749644.

31. Caton S, Chapman M (2016) The use of social media and people with intellectual disability: A systematic review and thematic analysis. Journal of Intellectual and developmental disability 41(2): 125-139.

32. Heitplatz VN, Bühler C, Hastall MR (2020) I Can't Do It, They Say!Perceived Stigmatization Experiences of People with Intellectual Disabilities When Using and Accessing the Internet. In International Conference on Human-Computer Interaction (pp. 390-408). Springer, Cham.

33. Chadwick DD, Fullwood C (2018) An online life like any other: identity, self-determination, and social networking among adults with intellectual disabilities. Cyberpsychology, Behavior, and Social Networking 21(1): 56-64.

\section{Your next submission with Juniper Publishers will reach you the below assets}

- Quality Editorial service

- Swift Peer Review

- Reprints availability

- E-prints Service

- Manuscript Podcast for convenient understanding

- Global attainment for your research

- Manuscript accessibility in different formats

( Pdf, E-pub, Full Text, Audio)

- Unceasing customer service

Track the below URL for one-step submission https://juniperpublishers.com/online-submission.php 